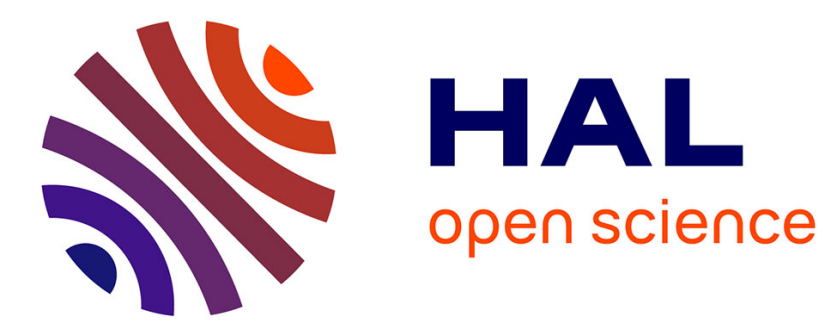

\title{
A quantum central limit theorem for sums of IID random variables
}

\author{
Vojkan Jaksic, Yan Pautrat, Claude-Alain Pillet
}

\section{To cite this version:}

Vojkan Jaksic, Yan Pautrat, Claude-Alain Pillet. A quantum central limit theorem for sums of IID random variables. Journal of Mathematical Physics, 2010, 51 (1), pp.015208. 10.1063/1.3285287 . hal-00423440

\section{HAL Id: hal-00423440 https://hal.science/hal-00423440}

Submitted on 9 Oct 2009

HAL is a multi-disciplinary open access archive for the deposit and dissemination of scientific research documents, whether they are published or not. The documents may come from teaching and research institutions in France or abroad, or from public or private research centers.
L'archive ouverte pluridisciplinaire HAL, est destinée au dépôt et à la diffusion de documents scientifiques de niveau recherche, publiés ou non, émanant des établissements d'enseignement et de recherche français ou étrangers, des laboratoires publics ou privés. 


\title{
A quantum central limit theorem for sums of IID random variables
}

\author{
V. Jakšić ${ }^{1}$, Y. Pautrat ${ }^{2}$, C.-A. Pillet ${ }^{3}$ \\ ${ }^{1}$ Department of Mathematics and Statistics \\ McGill University \\ 805 Sherbrooke Street West \\ Montreal, QC, H3A 2K6, Canada \\ ${ }^{2}$ Université Paris-Sud \\ Laboratoire de Mathématiques d'Orsay \\ 91405 Orsay Cedex, France \\ ${ }^{3}$ CPT, UMR 6207* \\ Université du Sud, Toulon-Var, B.P. 20132 \\ 83957 La Garde Cedex, France
}

October 9, 2009

\begin{abstract}
We formulate and prove a general central limit theorem for sums of independent identically distributed non-commutative random variables.
\end{abstract}

\section{Introduction}

The central limit theorem (CLT) for sums of independent identically distributed (IID) random variables is one of the most fundamental result in classical probability theory. Together with its various extensions, this result has found numerous applications to a wide range of problems in classical physics. To our knowledge, the first occurrences of quantum central limit theorems can be traced back to the works of Cushen and Hudson $[\mathrm{CH}]$ and Hepp and Lieb [HL1, HL2] in the early ' 70. Since then quantum CLTs have been developed and used in a variety of contexts not restricted to quantum physics. As a non exhaustive list of examples let us mention:

1. Equilibrium quantum statistical mechanics: fluctuations of macroscopic observables, fluctuation algebra ([GV],[GVV1] and subsequent works by the same authors, [Ma1, Ma2], see also Chapter 5 in [Pe]).

\footnotetext{
${ }^{*}$ CNRS, Université de Provence, Université de la Méditerranée, Université du Sud.
} 
2. Quantum field theory: Bose fields as scaling limits of free fields ([De]), maximal entropy characterization of quasi-free states $([\mathrm{St}])$.

3. Non-equilibrium quantum statistical mechanics: linear response theory ([GVV3]), Einstein's relations, current fluctuations in locally interacting Fermi gases ([JPP1]).

4. Graph theory: asymptotic spectral analysis of large graphs ([Ho, HO]).

5. Quantum information theory: quantum estimation and hypothesis testing ([Ha1, $\mathrm{Ha} 2])$.

6. Structure theory of von Neumann algebras: free probability ([VDN]).

7. Combinatorics: statistics of random words ([Ku1, Ku2]).

In this note we shall prove a general central limit theorem for sums of quantum (tensor) independent identically random variables which can be seen as an analytic extension of the algebraic CLT of Giri and von Waldenfels [GW]. Our main tool is a quantum version of the Lévy-Cramér continuity theorem recently proved in [JPP2].

This note is organized a follows: Section 2 is a brief review of the few basic concepts of quantum probability that are needed to formulate our result. In Section 3 we recall some well known facts about CCR-algebras and their quasi-free states. We formulate and discuss our main result in Section 4. Finally, Section 5 is devoted to proofs.

Acknowledments. V.J. thanks the CPT, where part of this work has been done. His research was partly supported by NSERC. The research of Y.P. and C.-A.P. was partly supported by Agence Nationale de la Recherche under grant ANR-09-BLAN-0098-01.

\section{Framework}

In this section we recall a few ideas from non-commutative probability theory. For more details, we refer to $[\mathrm{Me}, \mathrm{VDN}]$ and references therein.

Quantization is a process in which classical observables, i.e., real functions on a phase space, are replaced by self-adjoint operators on a Hilbert space. Similarly, in quantum probability the role of random variables is played by self-adjoint operators affiliated to some von Neumann algebra $\mathfrak{M}$ on a Hilbert space $\mathcal{H}$. Probability measures are replaced by normal states, i.e., positive weakly continuous linear functionals on $\mathfrak{M}$ such that $\omega(\mathrm{I})=1$. By the von Neumann spectral theorem, for any self-adjoint $A$ on $\mathcal{H}$, there exists a spectral measure (i.e. a projection-valued probability measure) $\xi^{A}$ such that

$$
(u, f(A) u)=\int f(a) \mathrm{d}\left(u, \xi^{A}(a) u\right),
$$

for all $u \in \mathcal{H}$ and for every bounded Borel function $f$ (see [RS] for more details on the von Neumann spectral theorem). In particular, the normality of $\omega$ implies that $\omega^{A}=\omega \circ \xi^{A}$ is a Borel probability measure on $\mathbb{R}$ and

$$
\omega(f(A))=\int f(a) \mathrm{d} \omega^{A}(a),
$$


for all bounded Borel functions $f$. It is then natural to call $\omega^{A}$ the law of the random variable $A$. Similarly, when one considers a commuting family $A_{1}, \ldots, A_{n}$ of selfadjoint operators, there exists a spectral measure $\xi^{A_{1}, \ldots, A_{n}}$ on $\mathbb{R}^{n}$ such that

$$
\left(u, f_{1}\left(A_{1}\right) \ldots f_{n}\left(A_{n}\right) u\right)=\int f_{1}\left(a_{1}\right) \ldots f_{n}\left(a_{n}\right) \mathrm{d}\left(u, \xi^{A_{1}, \ldots, A_{n}}\left(a_{1}, \ldots, a_{n}\right) u\right),
$$

for all $u \in \mathcal{H}$ and for any bounded Borel functions $f_{1}, \ldots, f_{n}$ and it is natural to call the Borel probability measure $\omega \circ \xi^{A_{1}, \ldots, A_{n}}$ on $\mathbb{R}^{n}$ the joint law of the $n$-tuple $\left(A_{1}, \ldots, A_{n}\right)$. Therefore, as long as one considers commuting families of random variables, quantum probability reduces to classical probability. However, as soon as one considers simultaneously two (or more) non-commuting random variables $A$ and $B$, the situation changes dramatically: in general, there exists no measure $\mu$ on $\mathbb{R}^{2}$ such that

$$
\omega(f(A) g(B))=\int f(a) g(b) \mathrm{d} \mu(a, b),
$$

for all bounded Borel functions $f$ and $g$ (see Theorem 2.2 in Chapter 3 of [Da]). In physical terms, this reflects the well known fact that two non-commuting observables cannot be measured simultaneously.

As already mentioned, this note focuses on the most basic form of CLT: the asymptotic law of sums of IID random variables. While independence is a well defined concept in classical probability, the non-commutativity of quantum random variables makes its translation to quantum probability ambiguous. Indeed, there are several possible nontrivial definitions of statistical independence in the quantum world. We shall be concerned here with the so called tensor or commutative independence. The corresponding IID setting is as follows.

Let $\mathfrak{N}$ be a von Neumann algebra and $\omega$ a normal state on $\mathfrak{N}$. We denote by

$$
\mathfrak{M}=\bigotimes_{t \in \mathbb{N}^{*}}^{(\omega)} \mathfrak{N}_{t}
$$

the tensor product of countably many copies $\mathfrak{N}_{t}$ of $\mathfrak{N}$ stabilized by the state $\omega$ and by $\omega^{\otimes \mathbb{N}^{*}}=\otimes_{t \in \mathbb{N}^{*}} \omega$ the corresponding product state on $\mathfrak{M}$ (see e.g., [BR1], Section 2.7.2).

We identify any $A \in \mathfrak{N}$ with the corresponding element $A \otimes \mathrm{I} \otimes \mathrm{I} \cdots$ of $\mathfrak{M}$ and denote by $\tau$ the right shift

$$
\tau\left(A_{1} \otimes A_{2} \otimes \cdots\right)=\mathrm{I} \otimes A_{1} \otimes A_{2} \cdots,
$$

on $\mathfrak{M}$. To any self-adjoint $A \in \mathfrak{N}$ we can in this way associate a sequence $\left(\tau^{t}(A)\right)_{t \in \mathbb{N}}$ of IID random variables. Indeed, for any $t \in \mathbb{N}$,

$$
\begin{aligned}
\omega^{\otimes \mathbb{N}^{*}}\left(f_{0}(A) f_{1}(\tau(A)) \cdots f_{t}\left(\tau^{t}(A)\right)\right) & =\omega\left(f_{0}(A)\right) \omega\left(f_{1}(A)\right) \cdots \omega\left(f_{t}(A)\right) \\
& =\int f_{0}\left(a_{0}\right) \cdots f_{t}\left(a_{t}\right) \mathrm{d} \omega^{A}\left(a_{0}\right) \cdots \mathrm{d} \omega^{A}\left(a_{t}\right),
\end{aligned}
$$

holds for all bounded Borel functions $f_{0}, \ldots, f_{t}$.

For any $A \in \mathfrak{N}$ we set

$$
\bar{A}^{t}=\frac{1}{\sqrt{t}} \sum_{s=1}^{t}\left(\tau^{s}(A)-\omega(A)\right) .
$$


For any bounded continuous function $f$, it follows from the classical CLT that

$$
\lim _{t \rightarrow \infty} \omega^{\otimes \mathbb{N}^{*}}\left(f\left(\bar{A}^{t}\right)\right)=\int f(a) \mathrm{d} N(a)
$$

where $N$ is the centered Gaussian measure of variance $\omega\left([A-\omega(A)]^{2}\right)$.

The situation becomes more intricate when considering a $n$-tuple $\left(A_{1}, \ldots, A_{n}\right)$ of noncommuting self-adjoint elements of $\mathfrak{N}$. In this case, a natural extension of the central limit theorem is concerned with the limiting behavior of

$$
\omega^{\otimes \mathbb{N}^{*}}\left(f_{1}\left({\overline{A_{k_{1}}}}^{t}\right) \cdots f_{m}\left({\overline{A_{k_{m}}}}^{t}\right)\right)
$$

as $t \rightarrow \infty$ for functions $f_{1}, \ldots, f_{m}$ in a suitable class and $k_{1}, \ldots k_{m} \in\{1, \ldots, n\}$.

\section{CCR algebras and quasi-free states}

Let $\mathfrak{A}$ be a real vector space equipped with an anti-symmetric, possibly degenerate bilinear form $\varsigma$. The CCR algebra over the pair $(\mathfrak{A}, \varsigma)$ is the $C^{*}$-algebra $\mathcal{W}$ generated by the family of elements $(W(A))_{A \in \mathfrak{A}}$ with the product and conjugation defined by

$$
W(A) W(B)=\mathrm{e}^{-\mathrm{i} \varsigma(A, B)} W(A+B), \quad W(A)^{*}=W(-A),
$$

for all $A, B \in \mathfrak{A}$ and equipped with the minimal regular norm (see [MSTV], or [Pe] for the case of non-degenerate $\varsigma$ ). We note for later reference that these elements satisfy the Weyl commutation relation

$$
W(A) W(B)=\mathrm{e}^{-2 \mathrm{i} \varsigma(A, B)} W(B) W(A) .
$$

In particular, the algebra $\mathcal{W}$ is commutative if and only if $\varsigma=0$.

If $Q$ is a positive semi-definite bilinear form on $\mathfrak{A}$ such that

$$
\varsigma(A, B)^{2} \leq Q(A, A) Q(B, B)
$$

for all $A, B \in \mathfrak{A}$, then the map

$$
\omega_{Q}(W(A))=\mathrm{e}^{-\frac{1}{2} Q(A, A)},
$$

uniquely extends to a state on $\mathcal{W}$, the so called quasi-free state generated by $Q$. Let $\left(\mathcal{H}_{Q}, \pi_{Q}, \Omega_{Q}\right)$ be the GNS representation of $\mathcal{W}$ associated to this state. Since the function $\left(t_{1}, \ldots, t_{n}\right) \mapsto \omega_{Q}\left(W\left(t_{1} A_{1}+\cdots+t_{n} A_{n}\right)\right)$ has an entire analytic extension to $\mathbb{C}^{n}$, there is a linear map $A \mapsto \varphi_{Q}(A)$ from $\mathfrak{A}$ to the self-adjoint operators on $\mathcal{H}_{Q}$ such that $\pi_{Q}(W(A))=\mathrm{e}^{\mathrm{i} \varphi_{Q}(A)}$. Moreover, $\Omega_{Q} \in \operatorname{Dom}\left(\varphi_{Q}\left(A_{1}\right) \cdots \varphi_{Q}\left(A_{n}\right)\right)$ for arbitrary $A_{1}, \ldots, A_{n} \in \mathfrak{A}$ and we shall consequently denote

$$
\omega_{Q}\left(\varphi_{Q}\left(A_{1}\right) \cdots \varphi_{Q}\left(A_{n}\right)\right) \equiv\left(\Omega_{Q}, \varphi_{Q}\left(A_{1}\right) \cdots \varphi_{Q}\left(A_{n}\right) \Omega_{Q}\right) .
$$

We shall also write

$$
\omega_{Q}\left(f_{1}\left(\varphi_{Q}\left(A_{1}\right)\right) \cdots f_{n}\left(\varphi_{Q}\left(A_{n}\right)\right)\right)=\left(\Omega_{Q}, f_{1}\left(\varphi_{Q}\left(A_{1}\right)\right) \cdots f_{n}\left(\varphi_{Q}\left(A_{n}\right)\right) \Omega_{Q}\right),
$$


for bounded Borel functions $f_{1}, \ldots, f_{n}$, i.e., denote $\omega_{Q}$ and its unique normal extension to the enveloping von Neumann algebra $\pi_{Q}(\mathcal{W})^{\prime \prime}$ by the same symbol.

For any integer $n$ let $\mathcal{P}_{n}$ be the set of all permutations $\pi$ of $\{1, \ldots, 2 n\}$ such that

$$
\pi(2 j-1)<\pi(2 j), \text { and } \pi(2 j-1)<\pi(2 j+1),
$$

for every $j \in\{1, \ldots, n\}$. The state $\omega_{Q}$ satisfies the Wick theorem

$\omega_{Q}\left(\varphi_{Q}\left(A_{1}\right) \cdots \varphi_{Q}\left(A_{n}\right)\right)=\left\{\begin{array}{cl}\sum_{\pi \in \mathcal{P}_{n / 2}} \prod_{j=1}^{n / 2} \omega_{Q}\left(\varphi_{Q}\left(A_{\pi(2 j-1)}\right) \varphi_{Q}\left(A_{\pi(2 j)}\right)\right), & \text { if } n \text { is even; } \\ 0, & \text { if } n \text { is odd, }\end{array}\right.$

where

$$
\omega_{Q}\left(\varphi_{Q}(A) \varphi_{Q}(B)\right)=Q(A, B)+\mathrm{i} \varsigma(A, B)
$$

for $A, B \in \mathfrak{A}$.

If $\mathcal{W}$ is commutative then, under the quasi-free state $\omega_{Q}$, the family of random variables $\left(\varphi_{Q}(A)\right)_{A \in \mathfrak{A}}$ is jointly Gaussian with mean zero and covariance

$$
\omega_{Q}\left(\varphi_{Q}(A) \varphi_{Q}(B)\right)=Q(A, B) .
$$

In this sense, the notion of quasi-free state is a natural extension of Gaussian measure in non-commutative probability.

\section{Results}

Let us now return to the setup of Section 2 and formulate our main result.

Given a normal state $\omega$ on the von Neumann algebra $\mathfrak{N}$, the real vector subspace $\mathfrak{A} \subset \mathfrak{N}$ generated by the family $A_{1}, \ldots, A_{n}$ of (not necessarily distinct) self-adjoint elements of $\mathfrak{N}$ comes equipped with a natural anti-symmetric bilinear form, namely

$$
\varsigma(A, B)=\frac{1}{2 \mathrm{i}} \omega([A, B]),
$$

and a positive semi-definite bilinear form

$$
Q(A, B)=\operatorname{Re} \omega((A-\omega(A))(B-\omega(B)))=\operatorname{Re} \omega(A B)-\omega(A) \omega(B) .
$$

Setting $L(A, B)=\omega((A-\omega(A))(B-\omega(B)))$ for $A, B \in \mathfrak{N}$, we note that $(A, B) \mapsto$ $L\left(A^{*}, B\right)$ is a Hermitian form on $\mathfrak{N}$ such that

$$
Q(A, B)=\operatorname{Re} L\left(A^{*}, B\right), \quad Q(A, A)=L\left(A^{*}, A\right), \quad \varsigma(A, B)=\operatorname{Im} L\left(A^{*}, B\right),
$$

for all $A, B \in \mathfrak{A}$. The Cauchy-Schwarz inequality for this Hermitian form implies

$$
\varsigma(A, B)^{2} \leq\left|L\left(A^{*}, B\right)\right|^{2} \leq L\left(A^{*}, A\right) L\left(B^{*}, B\right)=Q(A, A) Q(B, B),
$$

for all $A, B \in \mathfrak{A}$. Hence, $Q$ generates a quasi-free state $\omega_{Q}$ on the CCR algebra $\mathcal{W}$ over $(\mathfrak{A}, \varsigma)$. With the notations introduced in the previous sections, one has 
Theorem 4.1 Let $\omega$ be a normal state on the von Neumann algebra $\mathfrak{N}$. The central limit theorem holds for any family $A_{1}, \ldots, A_{n}$ of self-adjoint elements of $\mathfrak{N}$ in the following sense.

(i) For any non-commutative polynomial $P$ in $n$ variables

$$
\lim _{t \rightarrow \infty} \omega^{\otimes \mathbb{N}^{*}}\left(P\left[{\overline{A_{1}}}^{t}, \ldots,{\overline{A_{n}}}^{t}\right]\right)=\omega_{Q}\left(P\left[\varphi_{Q}\left(A_{1}\right), \ldots, \varphi_{Q}\left(A_{n}\right)\right]\right) .
$$

(ii) For any bounded continuous functions $f_{1}, \ldots, f_{m}$ and any $k_{1}, \ldots, k_{m} \in\{1, \ldots, n\}$,

$$
\lim _{t \rightarrow \infty} \omega^{\otimes \mathbb{N}^{*}}\left(f_{1}\left({\overline{A_{k_{1}}}}^{t}\right) \cdots f_{m}\left({\overline{A_{k_{m}}}}^{t}\right)\right)=\omega_{Q}\left(f_{1}\left(\varphi_{Q}\left(A_{k_{1}}\right)\right) \cdots f_{m}\left(\varphi_{Q}\left(A_{k_{m}}\right)\right)\right) .
$$

(iii) For any bounded Borel functions $f_{1}, \ldots, f_{m}$ and any $k_{1}, \ldots, k_{m} \in\{1, \ldots, n\}$,

$$
\lim _{t \rightarrow \infty} \omega^{\otimes \mathbb{N}^{*}}\left(f_{1}\left({\overline{A_{k_{1}}}}^{t}\right) \cdots f_{m}\left({\overline{A_{k_{m}}}}^{t}\right)\right)=\omega_{Q}\left(f_{1}\left(\varphi_{Q}\left(A_{k_{1}}\right)\right) \cdots f_{m}\left(\varphi_{Q}\left(A_{k_{m}}\right)\right)\right),
$$

provided one of the two following conditions is satisfied for each $j \in\{1, \ldots, m\}$ :

1. $Q\left(A_{j}, A_{j}\right)>0$ and the set of discontinuity points of the function $f_{j}$ has zero Lebesgue measure.

2. $Q\left(A_{j}, A_{j}\right)=0$ and $f_{j}$ is continuous at zero.

Part (i) is the Giri-von Waldenfels algebraic CLT (Theorem 1 in [GW]). The GoderisVerbeure-Vets CLT (Theorem 1 in [GVV2]) is a special case of part (ii), obtained by setting $n=m=1, k_{1}=1$ and $f_{1}(x)=\mathrm{e}^{\mathrm{i} x}$. The first step in our proof is a multi-variate extension of this result (Lemma 5.1 below), i.e., convergence of pseudocharacteristic functions.

As already mentioned in the previous section, the limiting CCR-algebra $\mathcal{W}$ is commutative and the quasi-free state $\omega_{Q}$ is a Gaussian measure $(\varsigma=0)$ if and only if the state $\omega$ is a trace on the subalgebra generated by $A_{1}, \ldots, A_{n}$, i.e., $\omega(B C)=\omega(C B)$ for all $B, C$ in this subalgebra. Under this additional assumption, part (ii) is identical with Theorem 2 in [Ku1]. However, it should be noticed that under this assumption Theorem 1 in [Ku1] gives a stronger CLT, namely

$$
\lim _{t \rightarrow \infty} \omega^{\otimes \mathbb{N}^{*}}\left(g\left(P\left[{\overline{A_{1}}}^{t}, \ldots,{\overline{A_{n}}}^{t}\right]\right)\right)=\omega_{Q}\left(g\left(P\left[\varphi_{Q}\left(A_{1}\right), \ldots, \varphi_{Q}\left(A_{n}\right)\right]\right)\right),
$$

for all bounded continuous function $g$ and any non-commutative polynomial $P$. To extend part (ii) and prove such a result for non-tracial $\omega$ remains an open problem. The main difficulty is related to the fact that, even though the moments of the random variable $P\left[{\overline{A_{1}}}^{t}, \ldots,{\overline{A_{n}}}^{t}\right]$ do converge to that of $P\left[\varphi_{Q}\left(A_{1}\right), \ldots, \varphi_{Q}\left(A_{n}\right)\right]$ by part (i), the law of the later under the state $\omega_{Q}$ is not determined by its moments if the degree of $P$ is larger than 4 . In contrast, it follows directly from part (ii) that

$$
\begin{aligned}
& \lim _{t \rightarrow \infty} \omega^{\otimes \mathbb{N}^{*}}\left(g\left(P\left[f_{1}\left({\overline{A_{1}}}^{t}\right), \ldots, f_{n}\left({\overline{A_{n}}}^{t}\right)\right]\right)\right) \\
& \quad=\omega_{Q}\left(g\left(P\left[f_{1}\left(\varphi_{Q}\left(A_{1}\right)\right), \ldots, f_{n}\left(\varphi_{Q}\left(A_{n}\right)\right)\right]\right)\right),
\end{aligned}
$$

for any bounded continuous functions $g, f_{1}, \ldots, f_{n}$ since the moments of

$$
P\left[f_{1}\left(\varphi_{Q}\left(A_{1}\right)\right), \ldots, f_{n}\left(\varphi_{Q}\left(A_{n}\right)\right)\right]
$$

under the state $\omega_{Q}$ define a unique law. 


\section{Proof}

The proof of Theorem 4.1 is organized in three steps. We start by proving convergence of multivariate "quasi-characteristic functions"

$$
\lim _{t \rightarrow \infty} \omega^{\otimes \mathbb{N}^{*}}\left(\mathrm{e}^{\mathrm{i} \alpha_{1} \overline{A_{1}} t} \cdots \mathrm{e}^{\mathrm{i} \alpha_{n} \overline{A_{n}}}\right)=\omega_{Q}\left(\mathrm{e}^{\mathrm{i} \alpha_{1} \varphi_{Q}\left(A_{1}\right)} \cdots \mathrm{e}^{\mathrm{i} \alpha_{n} \varphi_{Q}\left(A_{n}\right)}\right),
$$

for any self-adjoint $A_{1}, \ldots, A_{n} \in \mathfrak{N}$, uniformly for $\left(\alpha_{1}, \ldots, \alpha_{n}\right)$ in compact subsets of $\mathbb{C}^{n}$. Assertion (i) then follows from a simple complex variables argument. Finally we apply a Lévy-Cramér type result to get (ii) and (iii).

\section{Step 1: Quasi-characteristic functions}

Lemma 5.1 Le $\omega$ be a normal state on the von Neumann algebra $\mathfrak{N}$. Then, for any self-adjoint $A_{1}, \ldots, A_{n} \in \mathfrak{N}$ and all $\left(\alpha_{1}, \ldots, \alpha_{n}\right) \in \mathbb{C}^{n}$ one has

$$
\begin{aligned}
& \lim _{t \rightarrow \infty} \omega^{\otimes \mathbb{N}^{*}}\left(\mathrm{e}^{\mathrm{i} \alpha_{1} \overline{A_{1}} t} \cdots \mathrm{e}^{\mathrm{i} \alpha_{n} \overline{A_{n}}{ }^{t}}\right) \\
& \quad=\exp \left(-\frac{1}{2} \sum_{1 \leq j, k \leq n} Q\left(A_{j}, A_{k}\right) \alpha_{j} \alpha_{k}-\mathrm{i} \sum_{1 \leq j<k \leq n} \varsigma\left(A_{j}, A_{k}\right) \alpha_{j} \alpha_{k}\right),
\end{aligned}
$$

where $Q(A, B)=\operatorname{Re} \omega(A B)-\omega(A) \omega(B)$ and $\varsigma(A, B)=\operatorname{Im} \omega(A B)$. Moreover, the convergence is uniform with respect to $\left(\alpha_{1}, \ldots, \alpha_{n}\right)$ in any compact subset of $\mathbb{C}^{n}$.

Proof. (A simple adaptation of the proof of Theorem 3.1 in [GVV2].) Without loss of generality we may assume that $\omega\left(A_{j}\right)=0$ for all $j \in\{1, \ldots, n\}$. We first note that

$$
\begin{aligned}
& \frac{1}{2} \sum_{1 \leq j, k \leq n} Q\left(A_{j}, A_{k}\right) \alpha_{j} \alpha_{k}+\mathrm{i} \sum_{1 \leq j<k \leq n} \varsigma\left(A_{j}, A_{k}\right) \alpha_{j} \alpha_{k} \\
& =\frac{1}{4} \sum_{1 \leq j, k \leq n} \omega\left(A_{j} A_{k}+A_{k} A_{j}\right) \alpha_{j} \alpha_{k}+\frac{1}{2} \sum_{1 \leq j<k \leq n} \omega\left(A_{j} A_{k}-A_{k} A_{j}\right) \alpha_{j} \alpha_{k} \\
& =\frac{1}{2} \sum_{1 \leq k \leq n} \omega\left(A_{k}^{2}\right) \alpha_{k}^{2}+\sum_{1 \leq j<k \leq n} \omega\left(A_{j} A_{k}\right) \alpha_{j} \alpha_{k} .
\end{aligned}
$$

The result follows from the fact that

$$
\omega^{\otimes \mathbb{N}^{*}}\left(\mathrm{e}^{\mathrm{i} \alpha_{1} \overline{A_{1}}{ }^{t}} \cdots \mathrm{e}^{\mathrm{i} \alpha_{n} \overline{A_{n}}}\right)=\left[\omega\left(\mathrm{e}^{\mathrm{i} \alpha_{1} A_{1} / \sqrt{t}} \cdots \mathrm{e}^{\mathrm{i} \alpha_{n} A_{n} / \sqrt{t}}\right)\right]^{t}
$$

and the expansion

$$
\begin{aligned}
& \omega\left(\mathrm{e}^{\mathrm{i} \alpha_{1} A_{1} / \sqrt{t}} \cdots \mathrm{e}^{\mathrm{i} \alpha_{n} A_{n} / \sqrt{t}}\right) \\
& =\sum_{k_{1}, \ldots, k_{n} \in \mathbb{N}} \mathrm{i}^{k_{1}+\cdots+k_{n}} t^{-\left(k_{1}+\cdots+k_{n}\right) / 2} \frac{\alpha_{1}^{k_{1}} \cdots \alpha_{n}^{k_{n}}}{k_{1} ! \cdots k_{n} !} \omega\left(A_{1}^{k_{1}} \cdots A_{n}^{k_{n}}\right) \\
& =1-\frac{1}{t}\left[\frac{1}{2} \sum_{1 \leq k \leq n} \omega\left(A_{k}^{2}\right) \alpha_{k}^{2}+\sum_{1 \leq k<j \leq n} \omega\left(A_{k} A_{j}\right) \alpha_{k} \alpha_{j}\right]+t^{-3 / 2} R_{t},
\end{aligned}
$$


where the remainder $R_{t}$ satisfies

$$
\left|R_{t}\right| \leq \exp \left(\sum_{j=1}^{n}\left\|A_{j}\right\|\left|\alpha_{j}\right|\right)
$$

for $t \geq 1$.

\section{Step 2: Polynomials}

Lemma 5.2 Under the assumptions of Lemma 5.1 one has

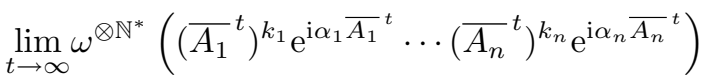

$$
\begin{aligned}
& =\omega_{Q}\left(\varphi_{Q}\left(A_{1}\right)^{k_{1}} \mathrm{e}^{\mathrm{i} \alpha_{1} \varphi_{Q}\left(A_{1}\right)} \cdots \varphi_{Q}\left(A_{n}\right)^{k_{n}} \mathrm{e}^{\mathrm{i} \alpha_{n} \varphi_{Q}\left(A_{n}\right)}\right),
\end{aligned}
$$

for all $k_{1}, \ldots, k_{n} \in \mathbb{N}$ and $\left(\alpha_{1}, \ldots, \alpha_{n}\right) \in \mathbb{C}^{n}$. In particular, Assertion (i) of Theorem 4.1 holds.

Proof. We set $\Gamma_{r}=\left\{z=\left(z_{1}, \ldots, z_{n}\right) \in \mathbb{C}^{n}|| z_{j} \mid=r\right.$ for all $\left.j\right\}$,

$$
F_{t}(z)=\omega^{\otimes \mathbb{N}^{*}}\left(\mathrm{e}^{\mathrm{i} z_{1} \overline{A_{1}} t} \cdots \mathrm{e}^{\mathrm{i} z_{n} \overline{A_{n}} t}\right)
$$

and

$$
F(z)=\exp -\left(\frac{1}{2} \sum_{1 \leq k \leq n} \omega\left(A_{k}^{2}\right) z_{k}^{2}+\sum_{1 \leq j<k \leq n} \omega\left(A_{j} A_{k}\right) z_{j} z_{k}\right) .
$$

As a byproduct of the proof of Lemma 5.1, we know that $F_{t}$ is an entire analytic function of $z \in \mathbb{C}^{n}$. By the Cauchy integral formula for polydisks, one has

$$
\frac{\partial^{k_{1}+\cdots+k_{n}} F_{t}}{\partial \alpha_{1}^{k_{1}} \cdots \partial \alpha_{n}^{k_{n}}}(\alpha)=\frac{k_{1} ! \cdots k_{n} !}{(2 \pi \mathrm{i})^{n}} \oint_{\Gamma_{r}} \frac{F_{t}(z)}{\left(z_{1}-\alpha_{1}\right)^{k_{1}+1} \cdots\left(z_{n}-\alpha_{n}\right)^{k_{n}+1}} \mathrm{~d} z_{1} \cdots \mathrm{d} z_{n},
$$

for all $\alpha \in D_{r}=\left\{\left(\alpha_{1}, \ldots, \alpha_{n}\right) \in \mathbb{C}^{n}|| \alpha_{j} \mid<r\right.$ for all $\left.j\right\}$. By Lemma 5.1, $F_{t}(z)$ converges uniformly to $F(z)$ on $\Gamma_{r}$ as $t \rightarrow \infty$. It follows that

$$
\lim _{t \rightarrow \infty} \frac{\partial^{k_{1}+\cdots+k_{n}} F_{t}}{\partial \alpha_{1}^{k_{1}} \cdots \partial \alpha_{n}^{k_{n}}}(\alpha)=\frac{\partial^{k_{1}+\cdots+k_{n}} F}{\partial \alpha_{1}^{k_{1}} \cdots \partial \alpha_{n}^{k_{n}}}(\alpha),
$$

and since $r$ can be made arbitrarily large, this holds for any $\alpha \in \mathbb{C}^{n}$. It remains to notice that

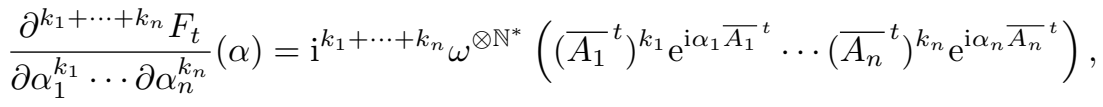

while

$$
\begin{aligned}
\frac{\partial^{k_{1}+\cdots+k_{n}} F}{\partial \alpha_{1}^{k_{1}} \cdots \partial \alpha_{n}^{k_{n}}}(\alpha) & =\frac{\partial^{k_{1}+\cdots+k_{n}}}{\partial \alpha_{1}^{k_{1}} \cdots \partial \alpha_{n}^{k_{n}}} \omega_{Q}\left(\mathrm{e}^{\mathrm{i} \alpha_{1} \varphi_{Q}\left(A_{1}\right)} \cdots \mathrm{e}^{\mathrm{i} \alpha_{n} \varphi_{Q}\left(A_{n}\right)}\right) \\
& =\mathrm{i}^{k_{1}+\cdots+k_{n}} \omega_{Q}\left(\varphi_{Q}\left(A_{1}\right)^{k_{1}} \mathrm{e}^{\mathrm{i} \alpha_{1} \varphi_{Q}\left(A_{1}\right)} \cdots \varphi_{Q}\left(A_{n}\right)^{k_{n}} \mathrm{e}^{\mathrm{i} \alpha_{n} \varphi_{Q}\left(A_{n}\right)}\right) .
\end{aligned}
$$


Step 3: Bounded continuous or Borel functions We shall now invoke a non-commutative version of the Lévy-Cramér continuity theorem proved in [JPP2]. To formulate this result in a form adapted to the present situation let us introduce some notation. We denote by $\mathcal{D}(f)$ the set of discontinuity points of a Borel function $f$. For fixed self-adjoint elements $A_{1}, \ldots, A_{n} \in \mathfrak{N}$ and for each $j \in\{1, \ldots, n\}, \alpha \in \mathbb{R}^{n}$ we define a Borel probability measure by

$\sigma_{j, \alpha}(f)=\omega_{Q}\left(\mathrm{e}^{\mathrm{i} \alpha_{1} \varphi_{Q}\left(A_{1}\right)} \cdots \mathrm{e}^{\mathrm{i} \alpha_{j-1} \varphi_{Q}\left(A_{j-1}\right)} f\left(A_{j}\right) \mathrm{e}^{-\mathrm{i} \alpha_{j-1} \varphi_{Q}\left(A_{j-1}\right)} \cdots \mathrm{e}^{-\mathrm{i} \alpha_{1} \varphi_{Q}\left(A_{1}\right)}\right)$.

In the notation of [JPP2], this measure is $\sigma_{j \infty}^{-}\left(\alpha_{1}, \ldots, \alpha_{j-1} ; \cdot\right)$ and Theorem 2 of [JPP2] becomes

Theorem 5.3 Let $\omega$ be a normal state on the von Neumann algebra $\mathfrak{N}$ and assume that for all $n \geq 1, \alpha \in \mathbb{R}^{n}$ and self-adjoint $A_{1}, \ldots, A_{n} \in \mathfrak{N}$ one has

$$
\lim _{t \rightarrow \infty} \omega\left(\mathrm{e}^{\mathrm{i} \alpha_{1} \overline{A_{1}} t} \cdots \mathrm{e}^{\mathrm{i} \alpha_{n} \overline{A_{n}} t}\right)=\omega_{Q}\left(\mathrm{e}^{\mathrm{i} \alpha_{1} \varphi_{Q}\left(A_{1}\right)} \cdots \mathrm{e}^{\mathrm{i} \alpha_{n} \varphi_{Q}\left(A_{n}\right)}\right) .
$$

Then, for any bounded Borel functions $f_{1}, \ldots, f_{n}$ one has

$$
\lim _{t \rightarrow \infty} \omega\left(f_{1}\left({\overline{A_{1}}}^{t}\right) \cdots f_{n}\left({\overline{A_{n}}}^{t}\right)\right)=\omega_{Q}\left(f_{1}\left(\varphi_{Q}\left(A_{1}\right)\right) \cdots f_{n}\left(\varphi_{Q}\left(A_{n}\right)\right)\right),
$$

provided $\sigma_{j \alpha}\left(\mathcal{D}\left(f_{j}\right)\right)=0$ for all $j \in\{1, \ldots, n\}$ and for all $\alpha \in \mathbb{R}^{n}$.

By Lemma 5.1, Assertion (ii) of Theorem 4.1 follows immediately. To prove Assertion (iii) we note that, by the Weyl commutation relation (3.2), the characteristic function of $\sigma_{j \alpha}$ is given by

$$
\begin{aligned}
\omega_{Q}\left(\mathrm{e}^{\mathrm{i} \alpha_{1} \varphi_{Q}\left(A_{1}\right)} \cdots\right. & \left.\mathrm{e}^{\mathrm{i} \alpha_{j-1} \varphi_{Q}\left(A_{j-1}\right)} \mathrm{e}^{\mathrm{i} \lambda \varphi_{Q}\left(A_{j}\right)} \mathrm{e}^{-\mathrm{i} \alpha_{j-1} \varphi_{Q}\left(A_{j-1}\right)} \cdots \mathrm{e}^{-\mathrm{i} \alpha_{1} \varphi_{Q}\left(A_{1}\right)}\right) \\
& =\omega_{Q}\left(\mathrm{e}^{\mathrm{i} \lambda \varphi_{Q}\left(A_{j}\right)}\right) \exp \left(2 \mathrm{i} \lambda \sum_{k=1}^{j-1} \varsigma\left(A_{j}, A_{k}\right) \alpha_{k}\right) \\
& =\exp \left(-\frac{\lambda^{2}}{2} Q\left(A_{j}, A_{j}\right)+2 \mathrm{i} \lambda \sum_{k=1}^{j-1} \varsigma\left(A_{j}, A_{k}\right) \alpha_{k}\right) .
\end{aligned}
$$

If $Q\left(A_{j}, A_{j}\right)>0$, it follows that $\sigma_{j \alpha}$ is Gaussian and hence absolutely continuous with respect to Lebesgue measure. If $Q\left(A_{j}, A_{j}\right)=0$, then (4.3) shows that $\varsigma\left(A_{j}, A_{k}\right)=0$ for all $k \in\{1, \ldots, n\}$. Then, $\sigma_{j \alpha}$ is Dirac measure at zero.

\section{References}

[BR1] Bratteli, O., Robinson, D. W.: Operator Algebras and Quantum Statistical Mechanics 1. Second edition, Springer, Berlin (1996).

[CH] Cushen, C.D., Hudson, R.L.: A quantum-mechanical central limit theorem. J. Appl. Prob. 8, 454 (1971).

[Da] Davies, E.B.: Quantum Theory of Open Systems. Academic Press, London (1976). 
[De] Dereziński, J.: Boson free fields as a limit of fields of a more general type. Rep. Math. Phys. 21, 405 (1985).

[GV] Goderis, D., Vets, P.: Central limit theorem for mixing quantum systems and the CCR algebra of fluctuations. Commun. Math. Phys. 122, 249 (1989).

[GVV1] Goderis, D., Verbeure, A., Vets, P.: About the mathematical theory of quantum fluctuations. In Mathematical Methods in Statistical Mechanics. Leuven Notes in Mathematical and Theoretical Physics. Series A: Mathematical Physics, 1. Leuven University Press, Leuven (1989).

[GVV2] Goderis, D., Verbeure, A., Vets, P.: Non-commutative central limits. Probab. Th. Rel. Fields 82, 527 (1989).

[GVV3] Goderis, D., Verbeure, A., Vets, P.: About the exactness of the linear response theory. Commun. Math. Phys. 136, 265 (1991).

[GW] Giri, N., von Waldenfels, W.: An algebraic version of the central limit theorem. Z. für Warscheinlichkeitstheorie 42, 129 (1978).

[Ha1] Hayashi, M.: Quantum estimation and the quantum central limit theorem. Preprint arXiv:quant-ph/0608198v1.

[Ha2] Hayashi, M.: Quantum Information. Springer, Berlin (2007).

[HL1] Hepp, K., Lieb, E.H.: Phase transitions in reservoir-driven open systems with applications to lasers and superconductors. Helv. Phys. Acta 46, 573 (1973).

[HL2] Hepp, K., Lieb, E.H.: On the superradiant phase transition for molecules in a quantized radiation field: The Dicke Maser model. Ann. Phys. 76, 360 (1973).

[Ho] Hora, A.: Central limit theorems and asymptotic spectral analysis on large graphs. Infin. Dimens. Anal. Quantum Probab. Relat. Top. 1, 221 (1998).

[HO] Hora, A., Obata, N.: Quantum Probability and Spectral Analysis of Graphs. Springer, Berlin (2007).

[JPP1] Jakšić, V., Pautrat, Y., Pillet, C.-A.: Central limit theorem for locally interacting Fermi gas. Commun. Math. Phys. 285, 175 (2009).

[JPP2] Jakšić, V., Pautrat, Y., Pillet, C.-A.: A non-commutative Lévy-Cramér continuity theorem, submitted.

[Ku1] Kuperberg, G.: A tracial quantum central limit theorem. Trans. Amer. Math. Soc. 357, 549 (2005).

[Ku2] Kuperberg, G.: Random words, quantum statistics, central limits, random matrices. Meth. and Appl. of Anal. 9, 99 (2002).

[Ma1] Matsui, T.: Bosonic central limit theorem for the one-dimensional XY model. Rev. Math. Phys. 14, 675 (2002).

[Ma2] Matsui, T.: On the algebra of fluctuation in quantum spin chains. Ann. Henri Poincaré 4, 63 (2003). 
[Me] Meyer, P.A.: Quantum probability for probabilists. Lecture Notes in Mathematics 1538, Springer, Berlin, (1993).

[MSTV] Manuceau, J., Sirugue, M., Testard, D., Verbeure, A.: The smallest $C^{*}$ algebra for canonical commutations relations. Commun. Math. Phys. 32, 231 (1973).

[Pe] Petz, D.: An invitation to the Algebra of Canonical Commutation Relations. Leuven Notes in Mathematical and Theoretical Physics. Series A: Mathematical Physics, 2. Leuven University Press, Leuven (1990).

[RS] Reed, M., Simon, B.: Methods of Modern Mathematical Physics, I. Functional Analysis. Academic Press, London (1980).

[Sp] Speicher, R.: A non-commutative central limit theorem. Math. Zeitschrift 209, 55 (1992).

[St] Streater, R.F.: Entropy and the central limit theorem in quantum mechanics. J. Phys. A 20, 4321 (1987).

[VDN] Voiculescu, D.V., Dykema, K.J., Nica, A.: Free Random Variables. CRM Monograph Series, AMS, Providence, RI (1992). 